\title{
Modeling the Black Hole Recoil from the Nucleus of M83
}

\author{
Guilherme G. Ferrari ${ }^{1}$, Horacio Dottori ${ }^{1}$, Rubén J. Díaz ${ }^{2}$ \\ ${ }^{1}$ Instituto de Física, Universidade Federal do Rio Grande do Sul, Porto Alegre, Brazil \\ ${ }^{2}$ ICATE-CONICET, San Juan, Argentina \\ Email: gg.ferrari@gmail.com, hdottori@gmail.com,rdiaz@gemini.edu
}

Received April 8, 2013; revised May 18, 2013; accepted June 23, 2013

Copyright (C) 2013 Guilherme G. Ferrari et al. This is an open access article distributed under the Creative Commons Attribution License, which permits unrestricted use, distribution, and reproduction in any medium, provided the original work is properly cited.

\begin{abstract}
GEMINI + GMOS and Chandra emission-line spectroscopy reveal that the Fanaroff-Riley II radio-source J133658.3295105 is a local object behind the barred-spiral galaxy M83 that is projected onto the galaxy's disk at about 60" from the galaxy's optical nucleus. J133658.3-295105 and its radiolobes are aligned with the optical nucleus of M83 and two other radio-sources neither of which are supernova remnants or HII regions. The optical nucleus of M83 is off-centered by 2.7 " $(\approx 60 \mathrm{pc})$ with regard to the kinematic center. Its mass is within the range $(1-4) \times 10^{6} \mathrm{M}_{\odot}$ and the velocity dispersion at its center points to a non-resolved mass concentration of $\leq 10^{6} \mathrm{M}_{\odot}$. In this paper we study the circumstances in which the radio source would have been ejected from the central region of M83. We analyze different types of collisions of binary and triple systems of super-massive black holes (SMBHs) by numerical simulations using a Post-Newtonian approximation of order $7 / 2\left(\sim 1 / \mathrm{c}^{7}\right)$. We developed an N-body code specially built to numerically integrate the Post-Newtonian equations of motion with a symplectic method. Numerical experiments show that the code is robust enough to handle virtually any mass ratio between particles and to follow the interaction up to a SMBH separation of three Schwarzschild radii. We show that within the current Post-Newtonian approximation, a scenario in which one of the three SMBHs suffers a slingshot-like kick is best suited to explain the ejection of J133658.3-295105, which simultaneously produces the recoil of the remaining BH pair, which drags together a subset of stars from the original cluster forming a structure that mimics the off-center optical nucleus of M83. The simulation parameters are tuned to reproduce the velocities and positions of J133658.3-295105 as well as the optical nucleus and the putative SMBH at its center.
\end{abstract}

Keywords: Galaxies; Nuclei; Dynamics; Black Holes Interaction; Kick-Off

\section{Introduction}

The presence of the $6.3 \mathrm{keV}$ Fe-K $\alpha$ emission constrained the distance of the Fanaroff-Riley II radio source J133658.3-295105 to the vicinity of M83 [1]. The source also shows $\mathrm{H} \alpha$ in emission receding with a radial velocity on the order of $100-150 \mathrm{~km} / \mathrm{sec}$ with respect to M83 [2]. The morphology of the radio emission distribution that protrudes along a line joining the optical nucleus of M83 and J133658.3-295105 [1,3] suggests that the compact object has been ejected from the optical nucleus $(\mathrm{ON})$, which is itself off-set with respect to the galaxy's kinematic center. The ON has a mass of $(1-4) \times 10^{6} \mathrm{M}_{\odot}[4,5]$ and it presents a velocity dispersion $[5,6]$ that is compatible with the presence of a super-massive black hole with $\mathrm{M}_{\mathrm{SMBH}} \leq 10^{6} \mathrm{M}_{\odot}$ or a spatially unresolved mass concentration located at its centre.

There is growing evidence that most galaxies host super-massive black holes (SMBHs) at their centers. The correlation between SMBH mass and bulge velocity dispersion in spiral galaxies $[7,8]$ points to a concurrent evolution of these systems in a hierarchical scenario of disk-galaxy formation [9]. A paradigmatic outcome of a merger of BHs is the recoil of the resulting $\mathrm{BH}[10,11]$ in response to the anisotropic emission of gravitational waves [11]. These results raise the questions on how a SMBH can grow at the center of a galaxy through BHpair fusion alone, and whether this kind of merger always leads to an amount of gravitational wave radiation that ejects the pair off the nucleus.

A triple-BH encounter extends the possible scenarios, because the ejection of one of the participant BHs may occur far in advance of the merger of the remaining two objects [12]. A triple-BH encounter seems to best explain the jet of M87 [13] and may also open up the possibility of explaining the double nucleus galaxy CID-42 [14].

We consider the by-product of the BHs interaction to be important for the case of M83. Consequently, as con- 
straints in the present scenario, we look at the ejection of $\mathrm{J} 133658.3-295105$, its velocity with respect to ON, its mass derived [1] from models based on black hole kinematics and emissivity [15], the offset position of ON, the presence of a putative $\mathrm{BH}$ at its center and the approximate mass of this $\mathrm{BH}$ [5]. We do not attempt at this modeling stage of our simulation, to reproduce either the double radio-sources' ejection which characterizes the object J133658.3-295105 as one of the kind FanaroffRiley II object, or the alignment of its three components with the projected direction of ejection from the optical nucleus. We analyze different types of collisions of binary and triple SMBHs through numerical simulations using a Post-Newtonian approximation of order $7 / 2\left(\sim 1 / c^{7}\right)$. For this purpose, one of us (GGF) developed an N-body code specially suited for numerically integrating the Post-Newtonian equations of motion. In Section 2 we present the numerical method and the equations of motion. In Section 3 we present numerical experiments with isolated binary and triple SMBHs. In Section 4 we present the N-body simulations of the SMBHs embedded in a cluster. Finally, in Section 5 we compare our results to the central region of M83.

\section{Numerical Methods}

In our approach, Post-Newtonian equations are employed in all interactions in which at least one $\mathrm{SMBH}$ is present, while interactions between individual stars are computed in Newtonian form with a softening length $\sim 1 / \mathrm{N}$, where $\mathrm{N}$ is the total number of particles. We use a symplectic integration method based on the time-transformed leapfrog scheme [16], modified to account for the correct time symmetry of the Post-Newtonian equations during the numerical solution. The time symmetry was accomplished by a semi-implicit trapezoidal rule, which results in only one (Newtonian and Post-Newtonian) force calculation per timestep, while keeping energy conservation within a difference of $\leq 10^{-12}$ relative to the initial value. The simulations ended when the particles reached separations of the order of 3 Schwarzschild radii or when the end-time was reached. We emphasize that the symplectic integrators respect the system's Hamiltonian structure, conserving the symplectic form itself.

\subsection{Post-Newtonian Equations of Motion}

The Post-Newtonian $(P N)$ equations are obtained from Itoh's works [17,18], who derived expressions for two body interactions only. Here, we propose a generalization of Itoh's formalism for N-body equations of motion:

$$
m_{i} \frac{\mathrm{d} \vec{v}_{i}}{\mathrm{~d} t}=-\sum_{j \neq i}^{n} \frac{G m_{i} m_{j}}{r_{i j}^{2}}\left[(1+A) \frac{\boldsymbol{r}_{i j}}{r_{i j}}+B \boldsymbol{v}_{i j}\right],
$$

where $A$ and $B$ contain the different $P N$ orders and are functions of $m_{i}, m_{j}, r_{i}, r_{j}, v_{i}, v_{j}$. $\boldsymbol{r}_{i j}=-\boldsymbol{r}_{j i}$ and $\boldsymbol{v}_{i j}=-\boldsymbol{v}_{j i}$, are the relative distance and velocity of particles $i$ and $j$.

$$
\begin{aligned}
A= & \frac{1}{c^{2}} A_{1 P N}+\frac{1}{c^{4}} A_{2 P N}+\frac{1}{c^{5}} A_{2.5 P N} \\
& +\frac{1}{c^{6}} A_{3 P N}+\frac{1}{c^{7}} A_{3.5 P N}+O\left(\frac{1}{c^{8}}\right) \\
B= & \frac{1}{c^{2}} B_{1 P N}+\frac{1}{c^{4}} B_{2 P N}+\frac{1}{c^{5}} B_{2.5 P N} \\
& +\frac{1}{c^{6}} B_{3 P N}+\frac{1}{c^{7}} B_{3.5 P N}+O\left(\frac{1}{c^{8}}\right)
\end{aligned}
$$

Because General Relativity (GR) is a non-linear theory, such approximation is not strictly valid. Nevertheless, the lack of a practical relativistic formulation for N-body simulations [19] led us to use the present set of equations. The $P N$ corrections are taken into account for all the timesteps, but they are only significant in close encounters involving at least one $\mathrm{BH}$. According to $[17,18], A$ and $B$ refers respectively to the static and kinetic parts of the equations of motion. Integer order corrections such as $1 P N, 2 P N$ and $3 P N$ cause pericenter advance in binary systems; nevertheless, they are conservative in the sense that they secularly preserve the orbit's semi-major axis. Half-integer order corrections $2.5 P N$ and $3.5 P N$ are responsible for the orbital energy dissipation resulting from the emission of gravitational waves. In the present simulations, the particles are point masses without spin. Spin effects should appear as spin-orbit coupling in order $1.5 P N$, spin-spin coupling in order $2 P N$ and in the terms of the reaction to gravitational radiation $2.5 P N$ and $3.5 P N$ [20]. Although successive $P N$ corrections are a conesquence of GR effects, the resulting equations of motion have to be interpreted in terms of Newtonian dynamics [20] that is, once a convenient coordinate system is chosen, the results can be expressed in terms of positions, velocities and accelerations and visualized as trajectories in an absolute Euclidean space. Nevertheless, as the equations of motion are intrinsically relativistic they must be Lorentz invariant, must hold the correct perturbbative limit given by the Schwarzschild's metric geodetic when one of the masses tends to zero and they must be conservative. In other words, the equations of motion must allow a Lagrangian or Hamiltonian formulation when radiation-reactions terms are switched off. We also remark that compared with Aarseth's 2.5PN formulation [21], which implements equations reduced to the center of mass, our Post-Newtonian equations allow free election of the coordinate system's origin.

\section{Numerical Experiments}

We first treated the Keplerian problem, which gives results similar to those obtained by [16], effectively obtain- 
ing energy conservation within the machine precision. In what follows we consider the onset of $P N$ corrections in the Keplerian problem.

\subsection{Keplerian Problem with Post-Newtonian Corrections}

We adopted units where the gravitational constant $\mathrm{G}=1$. We take $m_{1}=m_{2}=1$, the orbit eccentricity $e=0.9$ and semi-major axis $a=1.0526$ which corresponds to an initial relative distance $d_{0}=2$. We arbitrarily adopted a speed of light $c_{\text {light }}=16$. As a matter of comparison, a binary system with joint mass $M_{\text {binary }}=10^{6} \mathrm{M}_{\odot}$ and $d_{0}=1$ pc results in light velocity $c_{\text {light }} \approx 4571$ in the adopted units system. Figure 1 shows a $P N$-integration during 1000 orbital periods where only conservative terms were taken into account. The left panel shows that energy is conserved to a precision better than one part in $10^{10}$. This warrants that without radiation terms, the orbital semi-axis does not suffer secular variation, and that, as expected, the binary does not decay. The right panel shows the orbital precession for the first ten revolutions.

We then integrated the same system with the complete set of $P N$ equations until reaching a $\mathrm{BH}$ separation of three Schwarzschild radii $\left(R_{\mathrm{Sch}}\right)$. In Figure 2, we show the final phase of the orbital in-spiraling due to the gravitational radiation emission. This simulation compares quailtatively well with Boyle's numerical integration of general relativistic equations [22, his Figure 3]. In Figure 3 we show the evolution of the orbital separation against the period. The zoom in the right panel shows the spatial distance at which the $P N$ approximation loses validity. The cross shows a separation of three Schwarzschild radii, which ocurred when the simulation was interrupted.

\subsubsection{Gravitational Recoil of a SMBH Pair}

It has been determined [23] that the maximum velocity of recoil in a binary system occurs when the mass ratio is $q \approx$ 0.382 . We adopted this mass ratio and simulated two cases

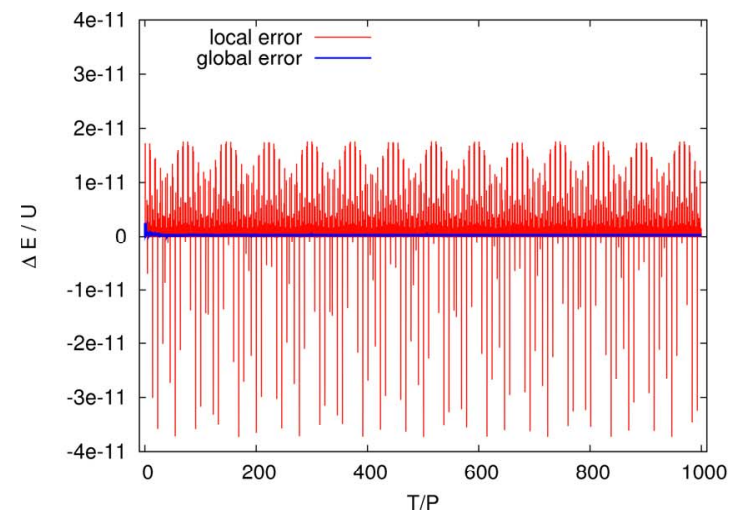

of binary gravitational recoil without spin, with eccentricities of $e=0$ and $e=0.9$. In both cases a total mass for the binary mass $M_{\text {binary }}=10^{7} \mathrm{M}_{\odot}$ was adopted. The initial distance between the binary components was given in each case by $d_{0}=2 \times 10^{-5} \mathrm{pc}$ for $e=0$ and $d_{0}=2 \times 10^{-4} \mathrm{pc}$ for $e=0.9$, which give $c_{\text {light }}=6.46$ and 20.44, respectively. This choice led to a similar time of coalescence in both cases. The upper panels in Figure 4 show the time evolution of the orbital separation for $e=0$ and $e=0.9$ while the lower panels show the center of mass recoil in a position vs. velocity diagram. The oscillation of the center of mass velocity is due to the continuous radiation of gravitational waves, which occurs in the direction opposite that of the orbital velocity. The amplitude of this oscillation is amplified according to the orbital eccentricity. The final plunges of both systems are practically similar.

\subsubsection{Gravitational Recoil of SMBH Triplets}

The chaotic nature of the three-body problem makes it practically impossible to sweep the whole set of parameters in triple-SMBH systems. We instead choose two systems with well known Newtonian evolution. Furthermore, these two systems differ greatly from the point of view of orbital stability. The first system is composed of three equal mass bodies, which move in an 8-like orbit. This system is extremely robust against small perturbations $[24,25]$ in Newtonian systems. The second system is the so-called Pythagorean system, in which masses with a ratio of 3:4:5 are located at the vertices of a traingle whose sides have sizes proportional to the mass located at the opposite vertex. We ran two simulations for each of these systems, and their parameters are shown in Table 1.

Model M111a corresponds to an 8-like orbit in which the intermediate body is initially at the center of mass. This model was integrated for a period of $\approx 1.05 \times 10^{10}$ years. After that period the $P N$ system differs slightly from the Newtonian system (Figure 5, left panel). Although 8-like systems are difficult to form [26] in mergers

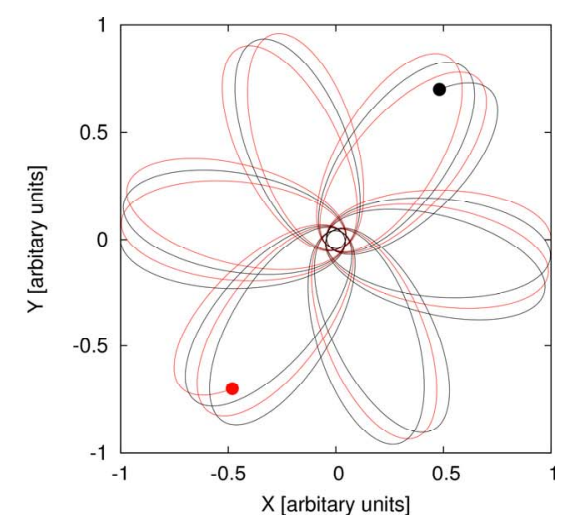

Figure 1. Left: energy conservation for a Keplerian binary $(e=0.9)$ integrated with conservative PN terms; Right: the orbital precession during the first ten orbits. 


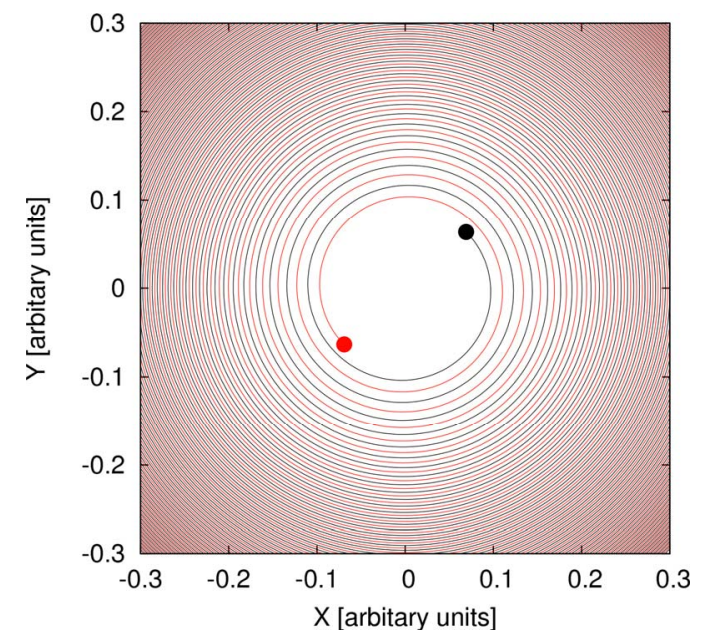

Figure 2. The spatial evolution of the $P N$ binary during the final phase due to the gravitational wave radiation.

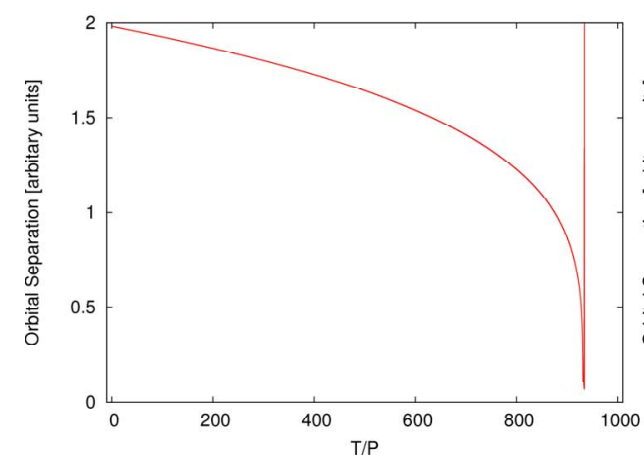

of galaxies, it would require efficient dynamical friction processes to coalesce them into a single black hole. Otherwise, these systems would end up as a triple black hole system and would survive as such for more than a Hubble time. If the system reaches a regime of strong gravitational radiation like the one illustrated with model M111b where masses and distances in model M111a have been multiplied by 100 and by 0.01 , respectively, the system coalescence would occur in less than 50 years. In this simulation the triple black hole starts to spiral inward, maintaining the 8-like configuration during the initial phase of the process until shortly before the coalescence of two of them, when the 8-like orbit major axis starts to precess rapidly due to the $P N$ effects. At this stage the simulation was interrupted, but the third black hole velocity components indicated that it would also merge with the other two into a single black hole. It is

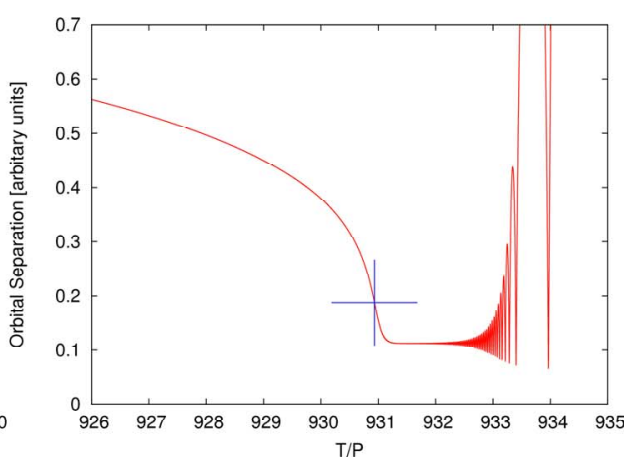

Figure 3. Left: separation of a PN binary circularly spiraling; Right: the moment in which the PN approximation lost validity; the + sign indicates a three $R_{\text {Sch }}$ separation.
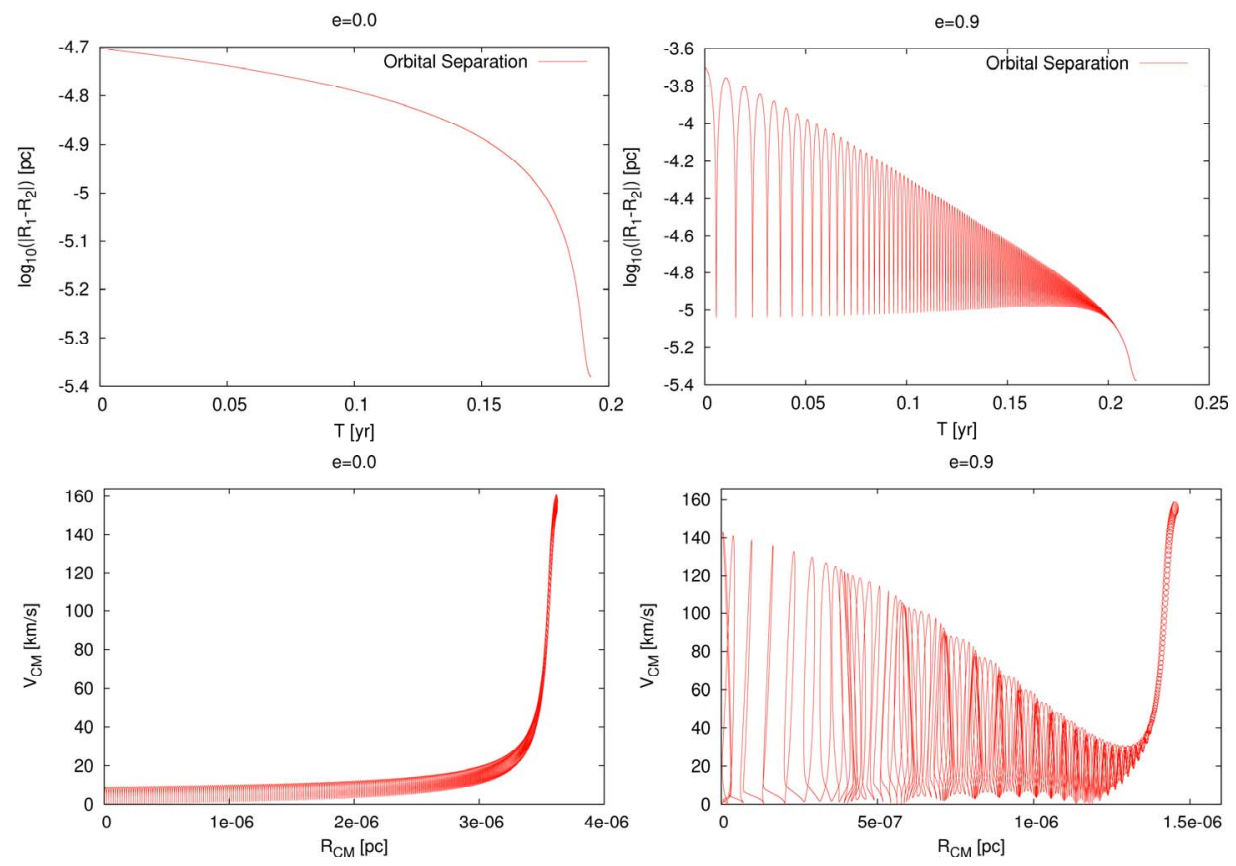

Figure 4. Time evolution of binary separation (upper panels) and center of mass position vs. velocity (lower panels) for $e=0$ (left) and $e=0.9$ (right). 

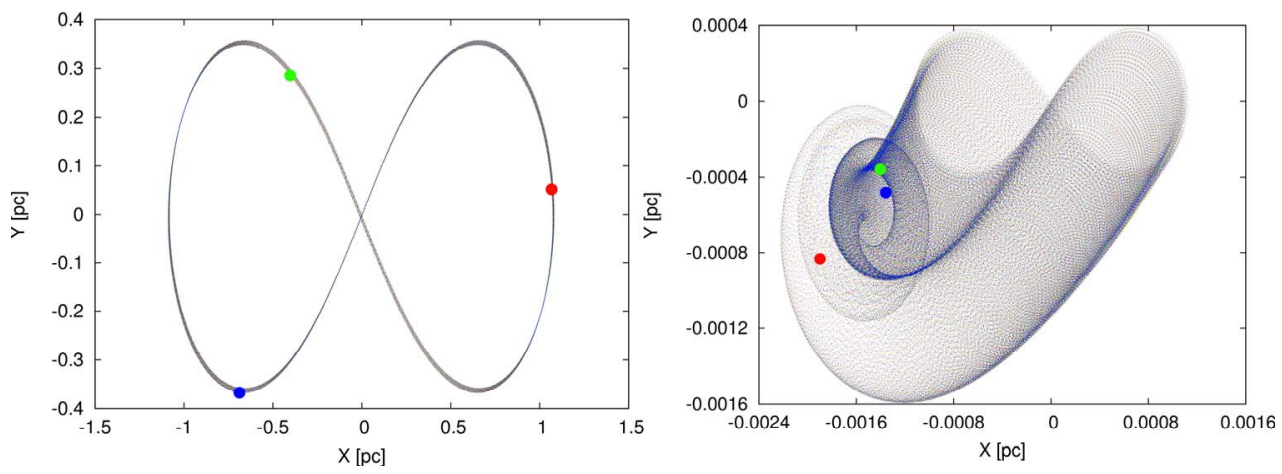

Figure 5. Left: orbital evolution during $1.05 \times 10^{10} \mathrm{yr}$ for model M111a. Right: orbital evolution of model M111b in which the SMBH's fusion occurs at $\approx \mathbf{4 4 . 4} \mathrm{yr}$.

Table 1. Columns: 1) system type; 2) model (numbers refer to mass ratio); 3) mass unit; 4) length unit; 5) speed of light in system velocity units, which is defined by choosing $[\mathrm{M}]$ and [L]).

\begin{tabular}{ccccc}
\hline System & Model & {$[\mathrm{M}]\left(\mathrm{M}_{\odot}\right)$} & {$[\mathrm{L}](\mathrm{pc})$} & $c_{\text {light }}$ \\
\hline \multirow{2}{*}{ 8-like configuration } & M111a & $10^{6}$ & 1 & $\approx 4571$ \\
& $\mathrm{M} 11 \mathrm{~b}$ & $10^{8}$ & 0.001 & $\approx 14.45$ \\
\multirow{3}{*}{ Pythagorean configuration } & $\mathrm{M} 345 \mathrm{a}$ & $10^{6}$ & 1 & $\approx 4571$ \\
& $\mathrm{M} 345 \mathrm{~b}$ & $10^{6}$ & 0.1 & $\approx 1445$ \\
\hline
\end{tabular}

worth noting that in spite of the three masses being equal, the gravitational recoil of the system is not inhibited, because the presence of a third mass produces an asymmetry in the emission of gravitational radiation.

Simulations of Pythagorean systems (models M345) are presented in Figure 6. These kinds of systems are more adequate for producing the ejection of the smaller black hole in time intervals much shorter than the Hubble time. Model M345a (Figure 6, upper left) initially evolves like the Newtonian case. Nevertheless, the orbits are deeply modified due to collisions with impact parameters on the order of tens to hundreds $R_{\mathrm{Sch}}$ until the smallest of the masses is kicked off from the system while the other two follow as a binary black hole in the opposite direction. Due to the strong eccentricity of the pair orbit, they reach the regime of strong gravitational radiation at pericenter encounters (Figure 6, upper right) and they finally merge in $\approx 7.38 \times 10^{4}$ years. The simulation was interrupted when the distance reached $3 R_{\text {Sch. }}$. The total simulation time was $5.27 \times 10^{5}$ years. The comparison of the binary black hole coalescence time with Peter's formulae [27] suggests an orbital eccentricity as high as $e$ $\approx 0.999-0.9999$. The velocity of the smallest black hole at the moment of ejection was $\approx 253 \mathrm{~km} / \mathrm{s}$ while the black hole pair recoils in the opposite direction at $\approx 84 \mathrm{~km} / \mathrm{s}$. Model M345b represents a system in which distances were diminished by a factor of ten (Figure 6, lower panels). In this model an almost frontal collision between the two more massive SMBHs leads to their coalescence in $\approx 7.1$ years.

\section{N-Body Simulations Including SMBHs}

\subsection{Observational Constraints}

The mass of $\mathrm{ON}$ has been estimated between $10^{6} \mathrm{M}_{\odot}$ and $(4 \pm 2) \times 10^{6} \mathrm{M}_{\odot}$ [5]. ON hosts a putative SMBH with a mass of $\leq 10^{6} \mathrm{M}_{\odot}[5,6]$. J133658.3-295105 is located at a projected distance of approximately $1 \mathrm{kpc}$ from $\mathrm{ON}$, receding with a radial velocity $V_{R} \approx 130 \mathrm{~km} / \mathrm{s}$ [2]. Using Fujita's model [15], the X-ray luminosity of J133658.3295105 was reproduced [1] with a SMBH mass on the order of $\approx 10^{6} \mathrm{M}_{\odot}$ moving at $V_{R} \approx 250 \mathrm{~km} / \mathrm{s}$ and an inclination of $30^{\circ}$ with respect to the galactic disk, which fits well the observed radial velocity of J133658.3295105.

In the next Section we model the whole system with the black holes embedded in a star cluster.

\subsection{N-Body Simulation of a SMBH Triplet Embedded in a Star Cluster}

We performed N-body simulations of a star cluster containing SMBHs to study the behavior of the system. Of particularly importance was finding out if we could reproduce the ejection of an object like J133658.3-295105 and its kinematics as well as find out if the position of the off-centered nucleus might be a consequence of the process of the SMBHs ejection. We constructed a spherecal distribution of equal masses according to a Hernquist profile [28] and an isotropic velocity distribution such that the system is in initial virial equilibrium. We followed prescriptions similar to those used by [29] for a Plummer profile. The experiments were performed by inserting different configurations of black holes and constraining their centers of mass to coincide with the cluster's potential center. In parallel, a rescaling was applied to the positions and velocities in order to re-establish the virial equilibrium of the whole (stars + SMBHs) system. In Table 2, we present the models for the SMBHs configurations M111 and M345. The parameter $c_{\text {light }}$ practically determines the SMBH system compactness, which 

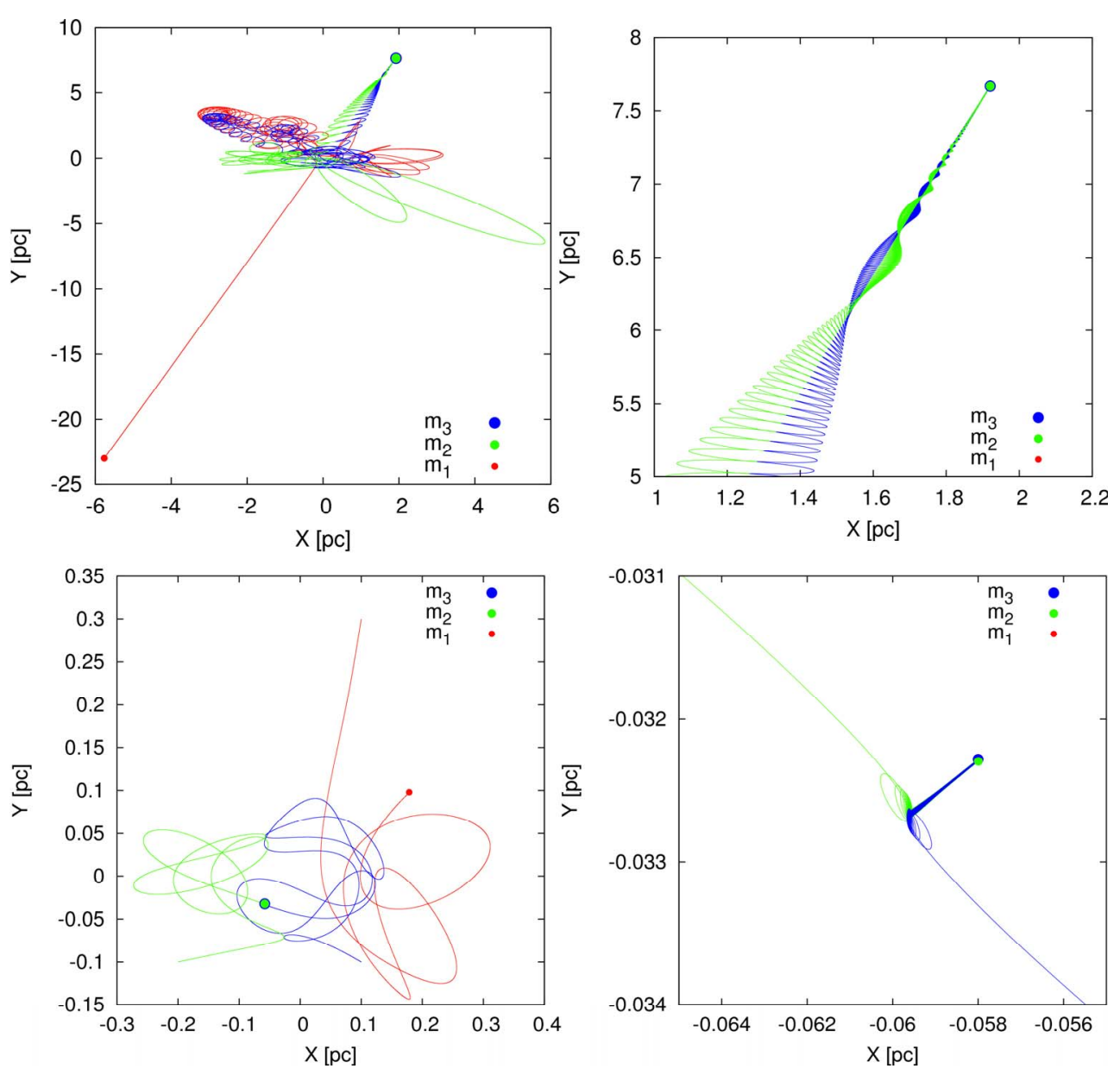

Figure 6. Upper panels: orbital evolution of model M345a. The simulation lasts $\approx 5.27 \times 10^{5}$ yr. Upper right: we detached the final coalescence phase of the more massive SMBHs pair, which took $7.38 \times 10^{4} \mathrm{yr}$. Lower panels: orbital evolution for $2646 \mathrm{yr}$ for model M345b. Lower right: final coalescence phase that took only $7.1 \mathrm{yr}$.

Table 2. Columns: 1) Model denomination (suffix a or b only refers to the random numbers sequence used in their construction); 2) Total number of particles (stars + 3 SMBHs); 3) Ratio between the SMBHs' masses and the cluster total mass (stars + 3 SMBHs); 4) Mass unit; 5) Length unit; 6) speed of light in system velocity units, which is defined by the choice of $[\mathrm{M}]$ and $[\mathrm{L}]$.

\begin{tabular}{cccccc}
\hline Model & $\mathrm{N}$ & $M_{\mathrm{BH}} / M_{\text {clus }}$ & {$[\mathrm{M}]\left(\mathrm{M}_{\odot}\right)$} & {$[\mathrm{L}](\mathrm{pc})$} & $c_{\text {light }}$ \\
\hline M111-4k-20a & 4096 & 0.2 & $10^{7}$ & 10 & $\sim 4571$ \\
M111-4k-20b & 4096 & 0.2 & $10^{7}$ & 10 & $\sim 4571$ \\
M111-4k-25a & 4096 & 0.25 & $8 \times 10^{6}$ & 10 & $\sim 5111$ \\
M111-4k-25b & 4096 & 0.25 & $8 \times 10^{6}$ & 10 & $\sim 5111$ \\
M111-8k-25 & 8192 & 0.25 & $8 \times 10^{6}$ & 10 & $\sim 5111$ \\
M345-4k-20a & 4096 & 0.2 & $10^{7}$ & 10 & $\sim 4571$ \\
M345-4k-20b & 4096 & 0.2 & $10^{7}$ & 10 & $\sim 4571$ \\
M345-4k-25a & 4096 & 0.25 & $8 \times 10^{6}$ & 10 & $\sim 5111$ \\
M345-4k-25b & 4096 & 0.25 & $8 \times 10^{6}$ & 10 & $\sim 5111$ \\
M345-8k-25 & 8192 & 0.25 & $8 \times 10^{6}$ & 10 & $\sim 5111$ \\
\hline
\end{tabular}

allows a comparison with the isolated SMBH systems quoted in Table 1 and discussed in the previous sections. For the present simulations, we adopted a precision parameter $\eta$ such that the energy error results are smaller than $10^{-6}$ throughout the process. The adopted softening parameter for the stars was $\varepsilon=4 / \mathrm{N}$.

Figure 7 shows the evolution of the central SMBHs in model M111-4k-25a. At approximately 1.4 Myr, two of the SMBHs are ejected, reaching distances on the order of $1 \mathrm{kpc}$ in a time $>10 \mathrm{Myr}$. The third SMBH remained in the cluster center. As a consequence of the ejection, the cluster swells up slightly. Figure 8 shows the evolution of model M345-8k-25. At approximately $0.8 \mathrm{Myr}$, one of the SMBHs is ejected, reaching distances on the order of $1 \mathrm{kpc}$ in approximately $20 \mathrm{Myr}$. The remaining pair became more bounded, showing that part of the ejection energy was gained to the expense of their potential energy. The binary SMBH migrates and drags out a part of the central cluster. The remainder of the cluster swells up significantly. The binary SMBH becomes increasingly bounded without reaching the strong gravitational radia- 


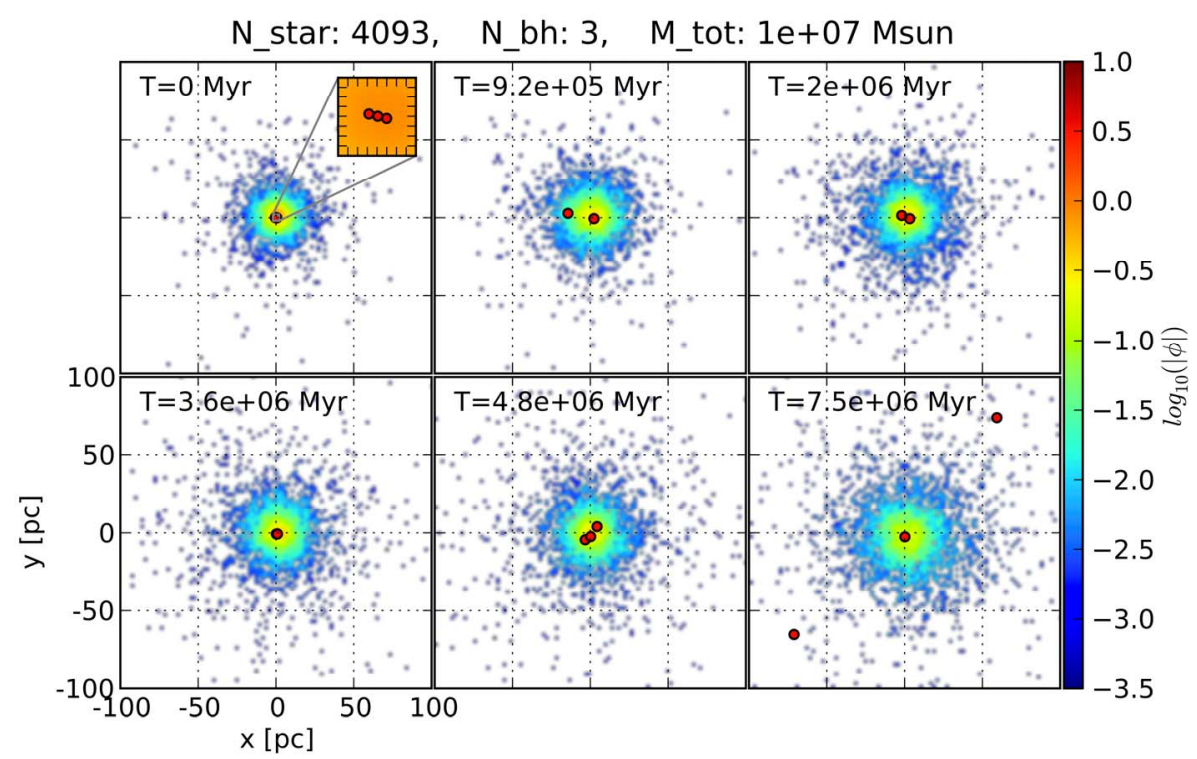

Figure 7. Snapshots of the evolution of the 8-like model M111-4k-25a. We show that two of the three equal mass SMBHs are ejected in opposite directions, leaving the third at the center of the distribution of stars that swell up slightly but otherwise remain centered at the original position.

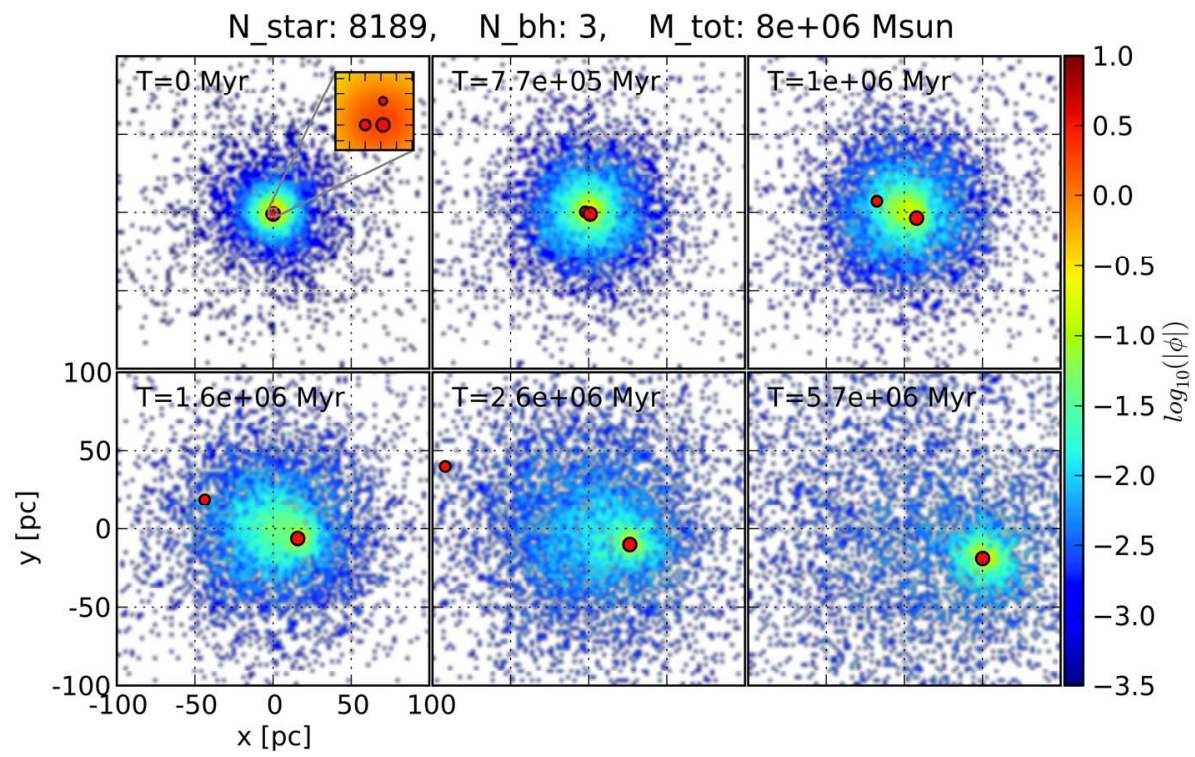

Figure 8. We show the evolution of the Pythagorean system M345-8k-25. The $M_{2}=4$ flies out, while the remaining pair $M_{1}+M_{3}$ $=8$ recoil more slowly, dragging a part of the embedding cluster.

tion regime in this period. It is worth noting that in this case the ejected SMBH was the $M_{2}=4$, and the SMBHpair that remained bound was the $M_{1}+M_{3}=8$. For all models the ejection of the isolated SMBH occured in approximately 0.1 to $2 \mathrm{Myr}$, reaching distances of 0.4 to 4 kpc until the end of the simulation.

Figure 9 shows the radial evolution of systems M111$4 \mathrm{k}-25 \mathrm{a}$ and M345-8k-25. The SMBH velocities at the ejection instant for the different models are presented in Table 3. We note that the drag out of a part of the central cluster only occurs if the velocity of the recoil of the
SMBH pair is up to about 1.5 times the initial velocity dispersion of the star cluster.

\section{Results and Discussion}

In this paper we extended Itoh's 3.5PN equations of motion to treat N-body problems. We study the behavior of 2-body and 3-body SMBH systems. For 3-body systems we studied two idealized configurations, namely, the 8like system, with three equal mass bodies and the Pythagorean system with masses in the ratio 3:4:5.

Within the current $P N$ approximation, the scenario with 

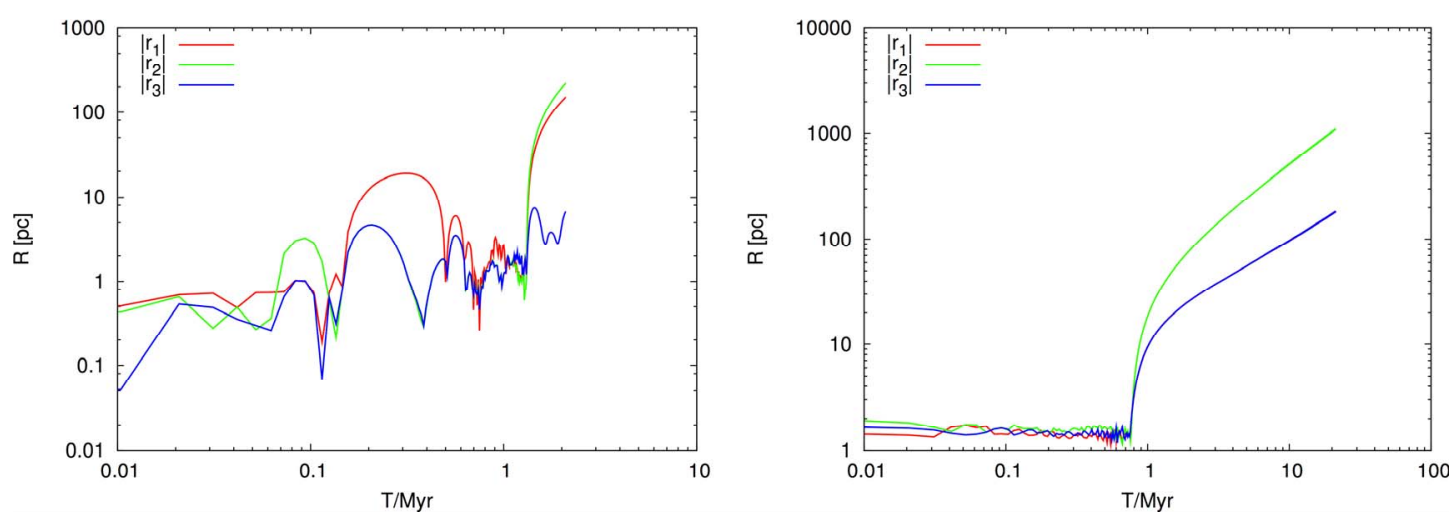

Figure 9. Time evolution of the radial position of each of the SMBHs for models M111-4k-25a (left) and M345-8k-25 (right).

Table 3. SMBH velocities at the moment of ejection for different models. Velocities are given in $\mathrm{km} / \mathrm{sec}$ and relative to the cluster's initial velocity dispersion $\sigma_{0}$. Presented velocities correspond to the remaining SMBH center of mass, and values in parenthesis correspond to the slingshotted SMBHs.

\begin{tabular}{cccccc}
\hline & M111-4k-20a & M111-4k-20b & M111-4k-25a & M111-4k-25b & M111-8k-25 \\
\hline$V(\mathrm{~km} / \mathrm{s})$ & $89(191)$ & $58(116)$ & $12(97,104)$ & $97(193)$ & $48(96)$ \\
$V / \sigma_{0}$ & $1.35(2.91)$ & $0.88(1.77)$ & $0.21(1.64,1.76)$ & $1.65(3.29)$ & $0.81(1.63)$ \\
& M345-4k-20a & M345-4k-20b & M345-4k-25a & M345-4k-25b & M345-8k-25 \\
$V(\mathrm{~km} / \mathrm{s})$ & $51(153)$ & $69(219)$ & $43(129)$ & $39(113)$ & $62(125)$ \\
$V / \sigma_{0}$ & $0.78(2.33)$ & $1.05(3.34)$ & $0.73(2.20)$ & $0.66(1.92)$ & $1.05(2.13)$ \\
\hline
\end{tabular}

three SMBHs is best suited to explain the ejection of a single SMBH. This kick-off reproduces well the projected present position and the motion of the object J133658.3295105 away from the ON. The displacement of part of the embedding cluster that accompanies the pair of remaining SMBH ranges over radial distances of $30-200 \mathrm{pc}$ on a timescale of $2 \times 10^{7} \mathrm{yr}$, which is in agreement with the on-the-sky projected distance of the ON from the kinematic center of M83 (60 pc). The accompanying cluster can be dragged out if the recoil velocity of the SMBH is less than $\sim 1.5$ times the initial velocity dispersion of the stars in the original cluster.

This simulation also furnishes a counterpart for the mass concentration located at the kinematical center of M83 [6], which is more extended than the ON [5]. This feature is produced in the model by the remains of the original star cluster, which swells up and stays centered at the former kinematical center position.

Our results show that in 3-body SMBH systems the recoil of an object like J133658.3-295105 may happen far in advance of the strong gravitational wave radiation regime.

Although the three SMBH models treated here are idealistic, it is easy to see that one can construct more realistic models that vary the mass ratios as well as their configurations. Though a wider range of configurations can be analyzed, we do not expect to achieve radically different results than those presented here.

\section{Acknowledgements}

GGF and HAD thank CNPq, Brazil, for financial support in the form of a fellowship and a research grant, respectively. RD acknowledges support from the SECYT at the National University of Cordoba and CONICET through PIP-0523. The computation was carried out at CESUPUFRGS, Brazil.

\section{REFERENCES}

[1] H. Dottori, R. Diaz, J. Albacete-Colombo and D. Mast, The Astrophysical Journal, Vol. 717, 2010, pp. L42-L46. doi: 10.1088/2041-8205/717/1/L42

[2] H. Dottori, R. Diaz and D. Mast, The Astronomical Journal, Vol. 136, 2008, pp. 2468-2472. doi:10.1088/0004-6256/136/6/2468

[3] L. Maddox et al., The Astronomical Journal, Vol. 132, 2006, pp. 310-320. doi:10.1086/505024

[4] D. Elmegreen, F. Chromey and A. Warren, The Astronomical Journal, Vol. 116, 1998, pp. 2834-2840. doi:10.1086/300657

[5] I. Rodrigues, H. Dottori, R. Díaz, M. Agüero and D. Mast, The Astronomical Journal, Vol. 137, 2009, pp. 40834090. doi:10.1088/0004-6256/137/5/4083

[6] N. Thatte, M. Tecza and R. Genzel, Astronomy \& Astro- 
physics, Vol. 364, 2000, pp. L47-L53.

[7] L. Ferrarese and D. Merrit, The Astrophysical Journal Letters, Vol. 539, 2000, pp. L9-L12. doi:10.1086/312838

[8] K. Gebhardt, et al., The Astrophysical Journal Letters, Vol. 539, 2000, pp. L13-L16. doi:10.1086/312840

[9] T. Okamoto, R. Nemmen and R. Bower, Monthly Notices of the Royal Astronomical Society, Vol. 385, 2008, pp. 161-181. doi:10.1111/j.1365-2966.2008.12883.x

[10] E. Bonning, G. Shields and S. Salviander, The Astrophysical Journal Letters, Vol. 666, 2007, pp. L13-L16. doi:10.1086/521674

[11] L. Blecha and A. Loeb, Monthly Notices of the Royal Astronomical Society, Vol. 390, 2006, pp. 1311-1325.

[12] M. Iwasawa, Y. Funato and J. Makino, The Astrophysical Journal, Vol. 651, 2006, pp. 1059-1067. doi:10.1086/507473

[13] D. Batcheldor, A. Robinson, D. Axon, E. Perlman and D. Merritt, The Astrophysical Journal Letters, Vol. 717, 2010, pp. L6-L10. doi:10.1088/2041-8205/717/1/L6

[14] F. Civano, et al., The Astrophysical Journal, Vol. 717, 2010, pp. 209-222. doi:10.1088/0004-637X/717/1/209

[15] Y. Fujita, The Astrophysical Journal, Vol. 691, 2009, pp. 1050-1057. doi:10.1088/0004-637X/691/2/1050

[16] S. Mikkola and S. Aarseth, Celestial Mechanics and Dynamical Astronomy, Vol. 84, 2002, pp. 343-354.

[17] Y. Itoh, Physical Review D, Vol. 69, 2004, Article ID: 064018 .
[18] Y. Itoh, Physical Review D, Vol. 80, 2009, Article ID: 124003.

[19] Y. Chu, Physical Review D, Vol. 79, 2009, Article ID: 044031.

[20] L. Blanchet, Living Reviews in Relativity, Vol. 9, 2006. doi:10.12942/1rr-2006-4

[21] S. Aarseth, Monthly Notices of the Royal Astronomical Society, Vol. 378, 2007, pp. 285-292. doi:10.1111/j.1365-2966.2007.11774.x

[22] M. Boyle, et al., Physical Review D, Vol. 76, 2007, Article ID: 124038.

[23] M. Fitchett, Monthly Notices of the Royal Astronomical Society, Vol. 203, 1983, pp. 1049-1062.

[24] C. Moore, Physical Review Letters, Vol. 70, 1993, pp. 3675-3679. doi:10.1103/PhysRevLett.70.3675

[25] A. Chenciner and R. Montgomery, Annals of Mathematics, Vol. 152, No. 3, 2000, pp. 881-901. doi: $10.2307 / 2661357$

[26] D. Heggie, Monthly Notices of the Royal Astronomical Society, Vol. 318, 2000, pp. L61-L63. doi:10.1046/j.1365-8711.2000.04027.x

[27] P. Peters, Physical Review, Vol. 136, 1964, pp. B1224B1232. doi:10.1103/PhysRev.136.B1224

[28] L. Hernquist, The Astrophysical Journal, Vol. 356, 1990, pp. 359-364. doi:10.1086/168845

[29] S. Aarseth, M. Henon and R. Wielen, Astronomy \& Astrophysics, Vol. 37, 1974, pp. 183-187. 